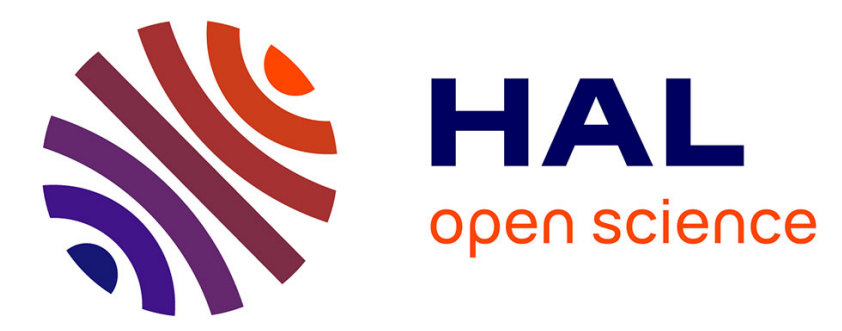

\title{
Antroduodénectomie avec anastomose gastroduodénale selon la technique de Péan pour ulcère duodénal peptique perforé
}

N Haythem, G. Godiris-Petit, S. Noullet, F. Menegaux, C. Trésallet, F. Varcusb

\section{To cite this version:}

N Haythem, G. Godiris-Petit, S. Noullet, F. Menegaux, C. Trésallet, et al.. Antroduodénectomie avec anastomose gastroduodénale selon la technique de Péan pour ulcère duodénal peptique perforé . Journal de Chirurgie Viscérale, 2017, 10.1016/j.jchirv.2017.05.002 . hal-01560029

\section{HAL Id: hal-01560029 \\ https://hal.sorbonne-universite.fr/hal-01560029}

Submitted on 11 Jul 2017

HAL is a multi-disciplinary open access archive for the deposit and dissemination of scientific research documents, whether they are published or not. The documents may come from teaching and research institutions in France or abroad, or from public or private research centers.
L'archive ouverte pluridisciplinaire HAL, est destinée au dépôt et à la diffusion de documents scientifiques de niveau recherche, publiés ou non, émanant des établissements d'enseignement et de recherche français ou étrangers, des laboratoires publics ou privés. 


\section{Titre :}

Antroduodénectomie avec anastomose gastroduodénale selon la technique de Péan pour ulcère duodénal peptique perforé.

\section{Auteurs :}

Haythem Najah ${ }^{1}$, Gaëlle Godiris-Petit ${ }^{1}$, Séverine Noullet ${ }^{1}$, Fabrice Menegaux ${ }^{1}$, Christophe Trésallet ${ }^{1}$, Florian Varcus ${ }^{2}$.

1- Service de chirurgie générale viscérale et endocrinienne, Hôpital de la PitiéSalpêtrière, 47-83, boulevard de l'Hôpital, Pierre-et-Marie-Curie-Sorbonne universités (Paris 6), 75013 Paris, France

2- Deuxième Département de chirurgie, clinique de chirurgie générale, digestive et endocrinienne, hôpital universitaire de Timisoara, Université de médecine Pharmacie Victor Babes, 2E Murgu Square, Timisoara, Roumanie.

\section{Auteur Correspondant :}

Pr Christophe Trésallet

christophe.tresallet@aphp.fr

Service de chirurgie générale viscérale et endocrinienne, Hôpital de la PitiéSalpêtrière, 47-83, boulevard de l'Hôpital, Pierre-et-Marie-Curie-Sorbonne universités, (Paris 6), 75013 Paris, France 
L'incidence de la pathologie ulcéreuse et de ses complications a considérablement régressée ces dernières années, grâce aux inhibiteurs de la pompe à protons et à la généralisation du traitement d'éradication de l'helicobacter pylori. Les indications de la chirurgie en urgence ont-elles aussi beaucoup diminuées grâce à la place grandissante de l'endoscopie et de la radiologie interventionnelle en cas d'hémorragie. En cas d'échec ou d'impossibilité d'hémostase, la chirurgie garde bien entendu toute sa place.

De plus, l'ulcère duodénal perforé reste cependant dans une majorité des cas une indication opératoire pour laquelle une suture simple par voie cœlioscopique représente le traitement de choix. Lorsque la perforation est large, avec une perte de substance duodénale importante, n'autorisant pas une suture à la fois efficace ou dans de bonnes conditions de sécurité, plusieurs techniques de réparation sont possibles. Chacune d'entre elles consiste à réaliser une antrectomie avec différentes possibilité de rétablissement de continuité digestive: anastomose gastrojéjunale manuelle sur toute (Polya) ou une partie (Finsterer) de la tranche gastrique, anastomose gastro-jéjunale mécanique latéro- latérale ou anastomose gastroduodénale (Péan).

L'anastomose gastroduodénale selon Péan présente de nombreux avantages dans les situations difficiles : d'éviter une suture duodénale peu sûre et de s'affranchir des fréquents problèmes d'incongruence entre l'estomac et le duodénum. Les problèmes de fermetures difficiles ou hasardeuses du moignon duodénal sont ainsi éliminés. [1]. 
Cette technique ancienne, réhabilitée par Dubois en 1971 [2], permet en rétablissant un circuit physiologique de diminuer le taux de fistules duodénales [3]. II s'agit d'une technique sure et efficace à long terme [4,5]

En dépit de la diminution de ses indications, la connaissance de cette technique nous paraît essentielle comme option chirurgicale de sauvetage des ulcères duodénaux compliqués.

Nous insistons ici sur la technique de Péan utilisée dans le cadre des pertes de substance non suturale du duodénum. La technique utilisée dans le cadre des hémorragies incontrôlables est rappelée [6].

Bibliographie :

[1] Fuerst EJ. Gastroduodenostomy. An answer to the difficult duodenal stump. Am J Surg 1968;115:287-90.

[2] Dubois F. Gastrectomy followed by gastroduodenal anastomosis for postbulbar ulcers and ulcers of the second portion of the duodenum. On 38 cases. J Chir (Paris) 1971;101:177-86.

[3] Kyzer S, Binyamini Y, Melki Y, Ohana G, Koren R, Chaimoff C, et al. Comparative study of the early postoperative course and complications in patients undergoing Billroth I and Billroth II gastrectomy. World J Surg 1997;21:763-766; discussion 767.

[4] Guinier D, Destrumelle N, Denue PO, Mathieu P, Heyd B, Mantion GA. Technique of antroduodenectomy without ulcer excision as a safe alternative treatment for bleeding chronic duodenal ulcers. World J Surg 2009;33:1010-4.

[5] Chereau N, Chandeze M-M, Tantardini C, Trésallet C, Lefevre JH, Parc Y, et al. Antroduodenectomy with Gastroduodenal Anastomosis: Salvage Emergency Surgery for Complicated Peptic Ulcer Disease--Results of a Double Institution Study of 35 Patients. J Gastrointest Surg Off J Soc Surg Aliment Tract 2016;20:539-45.

[6] Guinier D, Heyd D, Mantion G. Traitement chirurgical des ulcères duodénaux hémorragiques par antroduodénectomie sans excision de l'ulcère.J chir (paris) 2003 ; $140 ; 225-8$. 


\section{Figure 1 : Exposition - Bilan lésionnel.}

Sous anesthésie générale, le patient est installé en décubitus dorsal, les bras en croix. La voie d'abord de choix consiste a réaliser est une laparotomie médiane susombilicale, en général au décours d'une cœlioscopie qui n'a pas permis de traiter la perforation. Le lobe gauche du foie est récliné vers le haut afin d'exposer la région antro-pylorique.

Le schéma présente un ulcère duodénal perforé, large et sclérotique. Les caractéristiques de cet ulcère avec une large perte de substance, dont les berges sont fragiles et dont le siège est post pylorique distal (genu superius) rendent sa fermeture par suture simple hasardeuse.

Un ulcère duodénal postérieur hémorragique n'est bien évidemment pas accessible lors cette première exploration. On peut tout au plus deviner des caillots de sang à travers la paroi duodénale et gastrique.

\section{Figure 2 : Décollement duodénopancréatique de Kocher}

Un décollement duodénopancréatique (manœuvre de Kocher) est réalisé avant toute dissection de l'estomac. Cela d'obtenir une meilleure exposition de la région antropylorique, de pouvoir contrôler manuellement l'hémorragie en cas d'ulcère duodénal hémorragique associé et de préparer une anastomose gastro-duodénale sans 
tension. Le péritoine pariétal postérieur est incisé en dehors du $2^{\text {ème }}$ duodénum du genu superius et du genu inferius.

Le décollement est poursuivi jusqu'au bord gauche de veine cave inférieure et de la terminaison de la veine rénale gauche.

\section{Figure 3 : contrôle de l'hémorragie en cas d'ulcère duodénal hémorragique}

\section{associé [6]}

Les hémorragies incontrôlables sont souvent secondaires à une érosion de l'artère gastroduodénale par un ulcère duodénal postérieur creusant. Le trajet de cette artère est situé en arrière du bulbe duodénal. Une antro-pyloro-duodénotomie longitudinale permet d'évacuer les caillots de sang intragastrique, de préciser le siège exact et les caractéristiques de l'ulcère. L'hémostase est réalisée par des points appuyés de fils sertis non résorbables directement sur la zone hémorragique de l'artère, en veillant à ne pas prendre la voie biliaire principale dans son trajet rétro-duodénal. Le décollement de Kocher, précédemment réalisé, permet au chirurgien de soulever le bloc duodénopancréatique, facilitant ainsi cette étape et diminuant le risque de plaie cholédocienne.

\section{Figure 4 : Antroduodénectomie}

Les vaisseaux gastro-épiploiques droits et pyloriques sont liés au niveau de leur contact avec le duodénum. Le $1^{\text {er }}$ duodénum est libéré, si possible, jusqu'au genu superius. La grande courbure gastrique est libérée après section du ligament gastrocolique, en liant les branches épiploiques, depuis le bord inférieur du $1^{\text {er }}$ duodénum jusqu'à la zone avasculaire séparant les vaisseaux gastro-épiploiques et les 
vaisseaux courts. Le petit épiploon est effondré et les vaisseaux gastriques gauches sont liés au contact de l'estomac. L'estomac est sectionné grâce à l'application d'une rangée d'agrafes linéaires ménageant une bouche gastrique inférieure afin de réaliser l'anastomose gastroduodénale.

L'ulcère est emporté avec la pièce d'antro-duodénectomie (Figure 4A).

Dans le cas d'un ulcère duodénal hémorragique isolé (Figure 4B), la résection duodénale est plus limitée. La section du duodénum se fait à l'aplomb et juste au niveau du bord externe droit du cratère ulcéreux. Ceci permet d'éviter une dissection rétroduodénale pouvant exposer à des lésions biliaires ou pancréatiques.

\section{Figure 5 : Anastomose gastroduodénale - Mur postérieur}

L'anastomose gastro-duodénale, termino-terminale, est réalisée manuellement par des points séparés de fils lentement résorbables (types Vicryl@ $3 / 0$ ou 4/0). Pour la confection du mur postérieur, tous les points sont passés puis noués secondairement.

Lorsque le moignon duodénal est libre, les points prennent les séromusculeuses des parois gastrique puis duodénale (Figure 5A).

Dans le cas d'un ulcère hémorragique à fond scléreux, (Figure 5B), les points du mur postérieur prennent d'abord la séromusculeuse gastrique, s'appuient sur le bord externe droit du cratère scléreux en prenant la capsule pancréatique, pour sortir au contact du duodénum. Ainsi, la face postérieure de l'estomac vient se placer en ventouse contre le duodénum et exclure le cratère ulcéreux. 
Les points ne doivent pas être trop profond pour ne pas léser l'artère gastroduodénale ou le cholédoque. Au moindre doute, il ne faut pas hésiter à mettre une sonde tutrice dans le cholédoque pendant la réalisation de ce plan postérieur.

\section{Figure 6 : Anastomose gastroduodénale - Mur antérieur}

L'anastomose est terminée par la réalisation du mur antérieur, en veillant à assurer une parfaite étanchéité. Des points séparés de fils lentement résorbables $3 / 0$ ou 4/0 prennent les séromusculeuses des parois antérieures gastrique puis duodénale. Ces points sont d'abord passés puis noués secondairement. L'anastomose gastroduodénale doit être réalisée sans tension sur des tranches gastrique et duodénale congruentes et bien vascularisées. 